\title{
ALL EXPONENTIAL MOMENTS OF THE DYCK PATHS' MAXIMA (SCALED BY ROOT OF PATH LENGTH) ARE UNIFORMLY BOUNDED: AN ELEMENTARY PROOF
}

\author{
FELIX GRIMME
}

\begin{abstract}
Let $\mathcal{C}_{s}$ be the set of Dyck paths of length $2 s$. For all $s$ its cardinality is given by the Catalan number $C_{s}$, i.e., $\# \mathcal{C}_{s}=C_{s}$. We denote the maximum of a Dyck path $X$ by $X_{\max }$, and thereby we define the exponential moments $E_{s}(\lambda):=C_{s}^{-1} \sum_{X \in \mathcal{C}_{s}} \exp \left(\lambda(2 s)^{-1 / 2} X_{\max }\right)$, whereupon we scale the Dyck paths' maxima by the root of the path length. Then, there exists a function $d: \mathbb{R} \rightarrow \mathbb{R}$ so that $E_{s}(\lambda) \leq d(\lambda)$ for all $s \in \mathbb{N}$. We present an elementary proof of this wellknown result, which is based on the reflection principle and a telescoping sum trick; in particular, nothing about stochastic processes is used.
\end{abstract}

Introduction. In their groundbreaking work on the spectral edge of symmetric random matrices Sinai and Soshnikov use the result that all exponential moments of the Dyck paths' maxima are uniformly bounded, see [6, page 124]. In [5], Khorunzhiy and Marckert remark that the work of Sinai and Soshnikov lacks a bibliographical reference to this result. Khorunzhiy and Marckert present a proof, which relies on the work of Chung on Brownian excursions [2]. Kaigh, in turn, gives in his work [4] an elementary proof of Chung's result, utilizing a repeated reflection principle. In our work, this idea is combined with a telescoping sum trick to give an elementary proof of the result used by Sinai and Soshnikov.

Let $s \in \mathbb{N}_{0}$. A mapping $X:\{0, \ldots, 2 s\} \rightarrow \mathbb{N}_{0}$ fulfilling $X(0)=$ $X(2 s)=0$ and $|X(t)-X(t-1)|=1$ for all $1 \leq t \leq 2 s$ is called a Dyck path of length $2 s$. We set

$$
\mathcal{C}_{s}:=\{\text { Dyck paths of length } 2 s\} .
$$

2010 AMS Mathematics subject classification. Primary 60C05, 60G50.

Keywords and phrases. Dyck paths, exponential moments.

Received by the editors on February 23, 2014. 
In Stanley's book, [8, Exercise 6.19], one can find a list of combinatoric bijections from $\mathcal{C}_{s}$ to further 65 sets. Their common cardinality is given by the Catalan numbers

$$
C_{s}=\frac{1}{s+1}\left(\begin{array}{c}
2 s \\
s
\end{array}\right),
$$

which are ubiquitous in combinatorics. For Dyck paths $X \in \mathcal{C}_{s}$ we use the shorthand notation $X_{\max }:=\max _{0 \leq t \leq 2 s} X(t)$ for their maxima. For all $\lambda \in \mathbb{R}$, we define

$$
E_{s}(\lambda):=\frac{1}{C_{s}} \sum_{X \in \mathcal{C}_{s}} \exp \left(\lambda \frac{X_{\max }}{\sqrt{2 s}}\right) .
$$

Stochastically interpreted, these are the exponential moments of the random variable $X \mapsto X_{\max } / \sqrt{2 s}$ with respect to a uniform distribution on $\mathcal{C}_{s}$. In [5], Khorunzhiy and Marckert showed, amongst other things, that they are uniformly bounded in $s$ :

Theorem 1. There is a function $d: \mathbb{R} \rightarrow \mathbb{R}$ so that $E_{s}(\lambda) \leq d(\lambda)$ for all $s \in \mathbb{N}_{0}$.

As indicated before, Khorunzhiy and Marckert prove this result by tracing back the situation of Dyck paths to the situation of Brownian excursions, which had been studied earlier. The purpose of our paper is to give an elementary self-contained proof of this theorem.

A new elementary proof of Theorem 1. We divide the proof into four steps.

Step I. We set

$$
\mathcal{C}_{s}(x):=\left\{X \in \mathcal{C}_{s} \mid X_{\max }<x\right\}, \quad \text { and } \quad C_{s}(x):=\# \mathcal{C}_{s}(x),
$$

and we define the distribution function

$$
G_{s}(x):=P\left(\left\{X \in \mathcal{C}_{s} \mid X_{\max }<x\right\}\right)=\frac{C_{s}(x)}{C_{s}} .
$$

Apparently, it has the following properties:

(i) $G_{s}(x)=0$ for all $x \leq 0$,

(ii) $G_{s}(x)=1$ for all $x>s$,

(iii) $G_{s}$ is left-continuous, piecewise constant, and jumps at $0,1, \ldots, s$. 
Thus, we have

$$
\begin{aligned}
E_{s}(\lambda)= & \sum_{k=0}^{s} \exp \left(\lambda \frac{k}{\sqrt{2 s}}\right)\left(G_{s}(k+1)-G_{s}(k)\right) \\
= & \sum_{k=0}^{s} \exp \left(\lambda \frac{k}{\sqrt{2 s}}\right)\left(1-G_{s}(k)\right) \\
& -\sum_{k=1}^{s+1} \exp \left(\lambda \frac{k-1}{\sqrt{2 s}}\right)\left(1-G_{s}(k)\right) \\
= & \left(1-\exp \left(-\lambda \frac{1}{\sqrt{2 s}}\right)\right) \sum_{k=1}^{s} \exp \left(\lambda \frac{k}{\sqrt{2 s}}\right)\left(1-G_{s}(k)\right) \\
& +1-G_{s}(0)-\exp \left(\lambda \frac{s}{\sqrt{2 s}}\right)\left(1-G_{s}(s+1)\right) .
\end{aligned}
$$

Due to the above properties (i) and (ii) and $1-e^{-y} \leq y$, we get

$$
E_{s}(\lambda) \leq 1+\frac{\lambda}{\sqrt{2 s}} \sum_{k=1}^{s} \exp \left(\lambda \frac{k}{\sqrt{2 s}}\right)\left(1-G_{s}(k)\right)
$$

We define

$$
\widetilde{G}_{s}(k):=G_{s-1}(k-1) .
$$

To prove Theorem 1 , it is obviously enough to show for all $\lambda>0$ that

$$
\sup _{s \geq 2} \frac{\lambda}{\sqrt{s}} \sum_{k=2}^{s} e^{\lambda k / \sqrt{s}}\left(1-\widetilde{G}_{s}(k)\right)<\infty .
$$

Step II. We are looking for a useful expression for $\widetilde{G}_{s}$. We make a detour over more general random walks. A mapping $X:\{0, \ldots, p\} \rightarrow \mathbb{Z}$ fulfilling $X(0)=0, X(p)=q$ and $|X(t)-X(t-1)|=1$ for all $1 \leq t \leq p$ is called a random walk of type $(p, q)$. We set

$$
\mathcal{B}_{p, q}:=\{\text { random walks of type }(p, q)\} .
$$

For integers $a, b \in \mathbb{Z}$, we define furthermore

$$
\mathcal{B}_{p, q}(a, b):=\left\{X \in \mathcal{B}_{p, q} \mid a<\min _{t} X(t) \leq \max _{t} X(t)<b\right\} .
$$


Their cardinalities are denoted by $B_{p, q}:=\# \mathcal{B}_{p, q}$ and $B_{p, q}(a, b):=$ $\# \mathcal{B}_{p, q}(a, b)$. Thereby,

$$
\widetilde{G}_{s}(k)=\frac{C_{s-1}(k-1)}{C_{s-1}}=\frac{B_{2 s-2,0}(-1, k-1)}{C_{s-1}} .
$$

The next lemma, taken from the book by Billingsley ([1, page 78]), is crucial. For the sake of completeness its proof is in the appendix.

Lemma 2. Let $p \in \mathbb{N}_{0}$. For all integers $a, b, q$ with $a \leq 0 \leq b$ and $a<q<b$ the following formula holds:

$$
B_{p, q}(a, b)=\sum_{m \in \mathbb{Z}} B_{p, q+2 m(b-a)}-\sum_{m \in \mathbb{Z}} B_{p, 2 b-q+2 m(b-a)} .
$$

An immediate consequence of Lemma 2 is:

Corollary 3. For all integers $s \geq 1, k \geq 2$, we have

$$
\widetilde{G}_{s}(k)=1+2 \sum_{m \in k \cdot \mathbb{N}} \frac{s-2 m^{2}}{s+m} \prod_{j=1}^{m-1} \frac{s-j}{s+j} .
$$

Proof. We use (2) and the formula of Lemma 2.

$$
\begin{aligned}
C_{s-1} \cdot \widetilde{G}_{s}(k) & =B_{2 s-2,0}(-1, k-1) \\
& =\sum_{m \in \mathbb{Z}} B_{2 s-2,2 m k}-\sum_{m \in \mathbb{Z}} B_{2 s-2,2 k-2+2 m k}
\end{aligned}
$$

follows. After an index shift, we get

$$
\sum_{m \in \mathbb{Z}} B_{2 s-2,2 m k-2}
$$

for the second sum. Utilizing the symmetry $B_{p, q}=B_{p,-q}$, we obtain

$$
\begin{aligned}
C_{s-1} \cdot \widetilde{G}_{s}(k)= & B_{2 s-2,0}-B_{2 s-2,2} \\
& +\sum_{m=1}^{\infty}\left(2 B_{2 s-2,2 m k}-B_{2 s-2,2 m k-2}-B_{2 s-2,2 m k+2}\right) .
\end{aligned}
$$


We have $B_{p, q}=\left(\begin{array}{c}p \\ (p-q) / 2\end{array}\right)$, because random walks of type $(p, q)$ have to ascend $(p+q) / 2$ times and descend $(p-q) / 2$ times. The terms before the sum thus are

$$
B_{2 s-2,0}-B_{2 s-2,2}=\left(\begin{array}{c}
2 s-2 \\
s-1
\end{array}\right)-\left(\begin{array}{c}
2 s-2 \\
s-2
\end{array}\right)=\frac{1}{s}\left(\begin{array}{c}
2 s-2 \\
s-1
\end{array}\right)=C_{s-1} .
$$

For the summands with $s \geq m k+2$, we have

$$
\begin{aligned}
2 & B_{2 s-2,2 m k}-B_{2 s-2,2 m k-2}-B_{2 s-2,2 m k+2} \\
& =2\left(\begin{array}{c}
2 s-2 \\
s-m k-1
\end{array}\right)-\left(\begin{array}{c}
2 s-2 \\
s-m k
\end{array}\right)-\left(\begin{array}{c}
2 s-2 \\
s-m k-2
\end{array}\right) \\
& =\frac{(2 s-2) !\left(2 s-4(m k)^{2}\right)}{(s-m k) !(s+m k) !} \\
& =C_{s-1} \cdot 2 \frac{s-2(m k)^{2}}{s+m k} \frac{(s-1) !}{(s-m k) !} \frac{s !}{(s+m k-1) !} \\
& =C_{s-1} \cdot 2 \frac{s-2(m k)^{2}}{s+m k} \prod_{j=1}^{m k-1} \frac{s-j}{s+j}
\end{aligned}
$$

In the cases $s \leq m k-1, s=m k$, and $s=m k+1$ one checks directly that the first line of our calculation above equals the last. Successively, one obtains $0=0,-1=-1$ and $4-2 s=4-2 s$. Inserting our results and modifying the summation index we are done.

Step III. We define

$$
\pi_{m}:=\prod_{j=1}^{m} \frac{s-j}{s+j}
$$

The following observation (joint with Peter Otte, Ruhr-Universität Bochum) is the key to control the distribution function $\widetilde{G}_{s}$.

Lemma 4. Let $2 \leq k \leq s$ be integers. Then

$$
\sum_{m=k}^{s} \frac{2 m^{2}-s}{s+m} \pi_{m-1}=(k-1 / 2) \pi_{k-1} .
$$


Proof. Considered separately, the following two formulae,

$$
\frac{2 m^{2}-s}{s+m}=(m+1 / 2) \frac{2 m}{s+m}-1,
$$

and

$$
\pi_{m-1}-\pi_{m}=\pi_{m-1}\left(1-\frac{s-m}{s+m}\right)=\pi_{m-1} \frac{2 m}{s+m}
$$

are a matter of course. Surprisingly, putting them together yields

$\sum_{m=k}^{s} \frac{2 m^{2}-s}{s+m} \pi_{m-1}=\sum_{m=k}^{s}(m-1 / 2) \pi_{m-1}-(m+1 / 2) \pi_{m}=(k-1 / 2) \pi_{k-1}$,

where we used $\pi_{s}=0$ when evaluating the telescoping sum.

Step IV. To finish the proof of Theorem 1 we need the following three elementary estimates. Their proofs are in the appendix.

Proposition 5. Let $\left(a_{k}\right)_{k \in \mathbb{N}}$ be a sequence of real numbers with $a_{k}=0$ for almost all $k$, such that there exists a number $\xi \in \mathbb{N}$ with the following properties: $a_{k} \leq a_{k+1}$ if $k<\xi$, and $a_{k} \geq a_{k+1}$ if $k \geq \xi$. Then, for all $l \in \mathbb{N}:$

$$
\sum_{\substack{k=l \\ \text { step } l}}^{\infty} a_{k}:=\sum_{k \in l \cdot \mathbb{N}} a_{k} \leq a_{\max (l, \xi)}+\frac{1}{l} \sum_{k=l}^{\infty} a_{k} .
$$

(In Proposition 5 , the assumption $a_{k}=0$ for almost all $k$ is dispensable. The statement is even true for divergent series.)

Proposition 6. Let $a<\xi<b$ be real numbers, and let $f:[a, b] \rightarrow$ $[0, \infty)$ be monotonically increasing on $[a, \xi]$ and monotonically decreasing on $[\xi, b]$, respectively. Then the following estimate holds true:

$$
\sum_{k \in \mathbb{Z} \cap[a, b]} f(k) \leq f(\xi)+\int_{a}^{b} f(x) d x .
$$


Proposition 7. For all integers $m \geq 0$ and $n \geq 1$ the following holds:

$$
\prod_{k=1}^{m-1}\left(1-\frac{k}{n}\right) \leq \exp \left(-\frac{m^{2}}{2 n}+\frac{m}{2 n}\right)
$$

Corollary 3 yields

$$
\left(1-\widetilde{G}_{s}(k)\right) / 2=\sum_{m \in k \cdot \mathbb{N}} a_{m}
$$

where

$$
a_{m}=\frac{2 m^{2}-s}{s+m} \pi_{m-1}
$$

To make use of Proposition 5 we have to test $\left(a_{m}\right)_{m \in \mathbb{N}}$ for monotonicity. Immediately, we get $a_{m}=0$ if $m \geq s+1$, as 0 is a factor of the product $\pi_{m-1}$ in this case. If $m \leq s$, we have to find out about the sign of

$$
a_{m+1}-a_{m}=\left(\frac{2(m+1)^{2}-s}{s+m+1} \frac{s-m}{s+m}-\frac{2 m^{2}-s}{s+m}\right) \pi_{m-1} .
$$

The bracket can be written as

$$
\frac{\left(2(m+1)^{2}-s\right)(s-m)-\left(2 m^{2}-s\right)(s+m+1)}{(s+m)(s+m+1)} .
$$

The nominator of this fraction equals $(2 m+1)\left(3 s-2 m^{2}-2 m\right)$. It changes its sign exactly once, namely at $m_{0}=(-1+\sqrt{1+6 s}) / 2$ from plus to minus. The sequence $\left(a_{m}\right)_{m \in \mathbb{N}}$ thus complies with Proposition 5 applied to $\xi=\left\lceil m_{0}\right\rceil$. So, with Lemma 4 , we get

(3) $\frac{1}{\sqrt{s}} \sum_{k=2}^{s} e^{\lambda k / \sqrt{s}}\left(1-\widetilde{G}_{s}(k)\right) \leq \frac{2}{\sqrt{s}} \sum_{k=2}^{s} e^{\lambda k / \sqrt{s}}\left(a_{\max \left(\left\lceil m_{0}\right\rceil, k\right)}+\pi_{k-1}\right)$.

To further estimate $a_{\max \left(\left\lceil m_{0}\right\rceil, k\right)}$ we treat the cases $\max \left(\left\lceil m_{0}\right\rceil, k\right)=$ $\left\lceil m_{0}\right\rceil$ and $\max \left(\left\lceil m_{0}\right\rceil, k\right)=k$ separately. Firstly,

$$
a_{\left\lceil m_{0}\right\rceil} \leq \frac{2\left\lceil m_{0}\right\rceil^{2}-s}{s+\left\lceil m_{0}\right\rceil} \leq \frac{2\left(m_{0}+1\right)^{2}}{s}-1 \leq 5
$$

because $2\left(m_{0}+1\right)^{2}=1+\sqrt{1+6 s}+3 s \leq 6 s$ if $s \geq s_{0} \geq 2$. With the help of Proposition 7 and using $a_{k}=0$ for all $k \geq s+1$, we obtain 
$\pi_{k-1} \leq e \cdot e^{-k^{2} /(2 s)}$ and, therefore, secondly,

$$
a_{k}=\frac{2 k^{2}-s}{s+k} \pi_{k-1} \leq 2 e \frac{k^{2}}{s} e^{-k^{2} /(2 s)} .
$$

We insert this result into our inequality (3). Together with the estimates (4) and (5), we get

$$
\begin{aligned}
\frac{1}{\sqrt{s}} \sum_{k=2}^{s} e^{\lambda k / \sqrt{s}}\left(1-\widetilde{G}_{s}(k)\right) \\
\leq \frac{2}{\sqrt{s}} \sum_{k=2}^{\infty} e^{\lambda k / \sqrt{s}}\left(a_{\max \left(\left\lceil m_{0}\right\rceil, k\right)}+\pi_{k-1}\right) \\
\leq \frac{2}{\sqrt{s}}\left(\sum_{k=2}^{\left\lceil m_{0}\right\rceil} e^{\lambda k / \sqrt{s}}(5+1)+\sum_{k=\left\lceil m_{0}\right\rceil+1}^{\infty} e^{\lambda k / \sqrt{s}}\left(2 e k^{2} / s+e\right) e^{\left.-k^{2} /(2 s)\right)}\right. \\
\leq \frac{12}{\sqrt{s}}\left\lceil m_{0}\right\rceil e^{\lambda\left\lceil m_{0}\right\rceil / \sqrt{s}} \\
\quad+\frac{2 e}{\sqrt{s}} \sum_{k=1}^{\infty}\left(\frac{2 k^{2}}{s}+1\right) \exp \left(\lambda \frac{k}{\sqrt{s}}-\frac{k^{2}}{2 s}\right) .
\end{aligned}
$$

The $\left\lceil m_{0}\right\rceil$-term is uniformly bounded in $s$. We further estimate the rest:

$$
\begin{aligned}
\sum_{k=1}^{\infty}( & \left.\frac{2 k^{2}}{s}+1\right) \exp \left(\lambda \frac{k}{\sqrt{s}}-\frac{k^{2}}{2 s}\right) \\
\leq & \sum_{k=1}^{\lfloor\lambda \sqrt{s}\rfloor}\left(\frac{2 k^{2}}{s}+1\right) \exp \left(\lambda \frac{k}{\sqrt{s}}\right) \\
& +\sum_{k=\lceil\lambda \sqrt{s}\rceil}^{\infty}\left(\frac{2 k^{2}}{s}+1\right) \exp \left(-\frac{(k-\lambda \sqrt{s})^{2}}{2 s}+\frac{\lambda^{2}}{2}\right) \\
\leq & \lambda \sqrt{s}\left(2 \lambda^{2}+1\right) e^{\lambda^{2}} \\
& +e^{\lambda^{2} / 2} \sum_{k=\lceil\lambda \sqrt{s}\rceil}^{\infty}\left(\frac{2 k^{2}}{s}+1\right) \exp \left(-\frac{(k-\lceil\lambda \sqrt{s}\rceil)^{2}}{2 s}\right) .
\end{aligned}
$$

It remains to handle the summation over $k$. After an index shift, it 
equals

(8) $\sum_{k=0}^{\infty}\left(\frac{2(k+\lceil\lambda \sqrt{s}\rceil)^{2}}{s}+1\right) \exp \left(-\frac{k^{2}}{2 s}\right)$

$$
\leq c_{1} \sqrt{s}+c_{2} \sum_{k=\lceil\sqrt{s}\rceil}^{\infty} \frac{k^{2}}{s} \exp \left(-\frac{k^{2}}{2 s}\right)
$$

with constants $c_{1}$ and $c_{2}$, depending only on $\lambda$. The sum on the right hand side is in turn bounded from above by $\sum_{k=0}^{\infty} f(k)$ where $f(x)=x^{2} / s \exp \left(-x^{2} /(2 s)\right)$. The derivative

$$
f^{\prime}(x)=\frac{x}{s}\left(2-\frac{x^{2}}{s}\right) \exp \left(-\frac{x^{2}}{2 s}\right)
$$

is initially positive and then negative for $x>\sqrt{2 s}$. By Proposition 6 it follows for all $n \in \mathbb{N}$ that

$$
\sum_{k=0}^{n} f(k) \leq \frac{2}{e}+\sqrt{s} \int_{0}^{\infty} x^{2} e^{-x^{2} / 2} d x=\frac{2}{e}+\sqrt{s} \sqrt{\frac{\pi}{2}}
$$

and thus

$$
\sum_{k=\lceil\sqrt{s}\rceil}^{\infty} \frac{k^{2}}{s} \exp \left(-\frac{k^{2}}{2 s}\right) \leq \frac{2}{e}+\sqrt{s} \sqrt{\frac{\pi}{2}}
$$

Inserting the estimates (9) into (8), (8) into (7), and (7) into (6), we are done. Hence, Theorem 1 is proved.

\section{APPENDIX}

Proof of Lemma 2. To begin with, we remark that the sums in the formula claimed in Lemma 2 effectively are finite sums because $B_{p, q}=0$ for $|q|>p$.

Induction on $p$. First we check the formula for $p=0$. Due to

$$
B_{0, q}= \begin{cases}1 & \text { if } q=0 \\ 0 & \text { else }\end{cases}
$$


and $|2(b-a)|>|q|$, we have

$$
\sum_{m \in \mathbb{Z}} B_{0, q+2 m(b-a)}= \begin{cases}1 & \text { if } q=0, \\ 0 & \text { else. }\end{cases}
$$

Likewise, by means of $2 b-q \neq 0$ and $|2(b-a)|>|2 b-q|$,

$$
\sum_{m \in \mathbb{Z}} B_{0,2 b-q+2 m(b-a)}=0 .
$$

Induction step $p-1$ to $p$. For the first step of a path there are two possibilities: up or down. The rest of the path can be regarded as a path of its own, shorter by 1 . Thus, we have, firstly,

$$
B_{p, q}=B_{p-1, q-1}+B_{p-1, q+1}
$$

and, secondly,

$$
B_{p, q}(a, b)=B_{p-1, q-1}(a-1, b-1)+B_{p-1, q+1}(a+1, b+1) .
$$

Combining this with the induction hypothesis yields

$$
\begin{aligned}
B_{p, q}(a, b)= & \sum_{m \in \mathbb{Z}} B_{p-1, q-1+2 m(b-a)}-\sum_{m \in \mathbb{Z}} B_{p-1,2 b-q-1+2 m(b-a)} \\
& +\sum_{m \in \mathbb{Z}} B_{p-1, q+1+2 m(b-a)}-\sum_{m \in \mathbb{Z}} B_{p-1,2 b-q+1+2 m(b-a)} \\
= & \sum_{m \in \mathbb{Z}} B_{p, q+2 m(b-a)}-\sum_{m \in \mathbb{Z}} B_{p, 2 b-q+2 m(b-a)},
\end{aligned}
$$

which is the desired formula.

The proof above is indeed a simple way to check Lemma 2, but we do not learn where the formula originates. How to derive it from the reflection principle is set as a task in [1, Section 11], which is carried out in [3].

Proof of Proposition 5. Let $\widetilde{\xi} \in l \cdot \mathbb{N}$ such that $a_{\tilde{\xi}}=\max _{k \in l \cdot \mathbb{N}} a_{k}$. Since $\left(a_{k}\right)_{k}$ is piecewise monotonic, we have the estimate

$$
l \cdot \sum_{\substack{k=l \\ \text { step } l}}^{\infty} a_{k}=l a_{\tilde{\xi}}+\sum_{\substack{k=l \\ \text { step } l}}^{\tilde{\xi}-l} l a_{k}+\sum_{\substack{k=\tilde{\xi}+l \\ \text { step } l}}^{\infty} l a_{k}
$$




$$
\begin{aligned}
& \leq l a_{\tilde{\xi}}+\sum_{\substack{k=l \\
\text { step } l}}^{\tilde{\xi}-l}\left(a_{k}+a_{k+1}+\cdots+a_{k+l-1}\right) \\
& +\sum_{\substack{k=\tilde{\xi}+l \\
\text { step } l}}^{\infty}\left(a_{k}+a_{k-1}+\cdots+a_{k-l+1}\right) \\
& =l a_{\tilde{\xi}}+\sum_{k=l}^{\infty} a_{k}+\sum_{k=\tilde{\xi}+1}^{\infty} a_{k}=(l-1) a_{\tilde{\xi}}+\sum_{k=l}^{\infty} a_{k} .
\end{aligned}
$$

It is always true that $a_{\tilde{\xi}} \leq a_{\xi}$; in the case $l \geq \xi$, we even have $a_{\tilde{\xi}}=a_{l}$. Dividing by $l$ finishes the proof.

Proof of Proposition 6. At first, we remark that $f$ is piecewise continuous and thus integrable. The case $\mathbb{Z} \cap[a, b]=\emptyset$ is trivial. So, let $\mathbb{Z} \cap[a, b] \neq \emptyset$, and let $\widehat{\xi}$ be a point where $\left.f\right|_{\mathbb{Z} \cap[a, b]}$ takes its maximum. Then $\left.f\right|_{[k, k+1]} \geq f(k)$ for all $k \in \mathbb{Z} \cap[a, \widehat{\xi})$ and $\left.f\right|_{[k-1, k]} \geq f(k)$ for all $k \in \mathbb{Z} \cap(\widehat{\xi}, b]$. Hence, we have

$$
\begin{aligned}
\sum_{k \in \mathbb{Z} \cap[a, b]} f(k)= & f(\widehat{\xi})+\sum_{k \in \mathbb{Z} \cap[a, \hat{\xi})} f(k)+\sum_{k \in \mathbb{Z} \cap(\hat{\xi}, b]} f(k) \\
\leq & f(\widehat{\xi})+\sum_{k \in \mathbb{Z} \cap[a, \hat{\xi})} \int_{k}^{k+1} f(x) d x \\
& +\sum_{k \in \mathbb{Z} \cap(\hat{\xi}, b]} \int_{k-1}^{k} f(x) d x \\
\leq & f(\widehat{\xi})+\int_{a}^{\hat{\xi}} f(x) d x+\int_{\hat{\xi}}^{b} f(x) d x
\end{aligned}
$$

which implies our claim due to $f(\widehat{\xi}) \leq f(\xi)$.

Proof of Proposition 7. The case $m \geq n$ is trivial if $m \geq 2$. If $m=n=1$, both sides equal 1 . Thus, let $m<n$. Due to $\ln (1+x) \leq x$ for all $x>-1$, we have

$$
\ln \left(\prod_{k=1}^{m-1}\left(1-\frac{k}{n}\right)\right)=\sum_{k=1}^{m-1} \ln \left(1-\frac{k}{n}\right)
$$




$$
\leq-\sum_{k=1}^{m-1} \frac{k}{n}=-\frac{m(m-1)}{2 n},
$$

which implies the desired estimate.

\section{REFERENCES}

1. Patrick Billingsley, Convergence of probability measures, John Wiley \& Sons, Inc., New York, 1968.

2. K.L. Chung, Maxima in Brownian excursions, Bull. Amer. Math. Soc. 81 (1975), $742-745$.

3. Felix Grimme, Über eine Erweiterung der Methode von Soshnikov zur Untersuchung des größten Eigenwerts auf unsymmetrische Verteilungen, Ph.D. thesis, Universität Bayreuth, Germany, 2013.

4. W.D. Kaigh, An elementary derivation of the distribution of the maxima of Brownian meander and Brownian excursion, Rocky Mountain J. Math. 8 (1978), 641-645.

5. Oleksiy Khorunzhiy and Jean-François Marckert, Uniform bounds for exponential moment of maximum of a Dyck path, Electr. Comm. Prob. 14 (2009), 327-333.

6. Ya.G. Sină and A.B. Soshnikov, A refinement of Wigner's semicircle law in a neighborhood of the spectrum edge for random symmetric matrices, Funk. Anal. Pril. 32 (1998), 56-79, 96 (in Russian); Funct. Anal. Appl. 32 (1998), 114-131 (in English).

7. Richard P. Stanley, Enumerative combinatorics, Volume 1, Cambr. Stud. Adv. Math. 49, Cambridge University Press, Cambridge, 1997.

8. _ Enumerative combinatorics, Volume 2, Cambr. Stud. Adv. Math. 62, Cambridge University Press, Cambridge, 1999.

Oberer Grenzweg 20, 58454 Witten, Germany

Email address: felix.grimme@rub.de 